\title{
Some spherical solutions of ideal magnetohydrodynamic equations
}

\author{
PY PICARD ${ }^{a}$ \\ a Département de Physique, Université de Montréal, \\ C. P. 6128, Succ. Centre-ville, Montréal, (QC) H3C 3J7, Canada \\ E-mail: picardp@inbox.as
}

Received May 29, 200\%; Accepted in Revised Form August 24, 2007

\begin{abstract}
Some spherical solutions of the ideal magnetohydrodynamic (MHD) equations are obtained from the method of the weak transversality method (WTM), which is based on Lie group theory. This analytical method makes use of the symmetry group of the MHD system in situations where the "classical" Lie approach of symmetry reductions is no longer applicable. Also, a brief physical interpretation of these solutions is given.
\end{abstract}

\section{Lie group formalism for the ideal MHD equations.}

Efficient methods for obtaining exact solutions of systems of partial differential equations (PDEs) can be developed from Lie group theory. These methods have in common that they provide a reduction of the full set of PDEs to a reduced system which involves fewer independent variables. Before introducing the ideal magnetohydrodynamic (MHD) equations, let us briefly resume the application of symmetry for solving differential equations. For a more detailed review of this subject, see refs. [1]-[7].

Consider the following $k$-order system of $m$ PDEs

$$
\Delta^{l}\left(\mathrm{x}, \mathrm{u}^{(k)}\right)=0, \quad l=1, \ldots, m,
$$

involving $p$ independent variables $\mathrm{x}=\left(x_{1}, \ldots, x_{p}\right) \in E$ and $q$ dependent variables $\mathrm{u}=\left(u_{1}, \ldots, u_{q}\right) \in U$, and $\mathrm{u}^{(k)}$ denotes all partial derivatives of $\mathrm{u}$ up to order $k$. The numbers $p, q, k$ and $m$ are all nonnegative integers. Suppose that the local group $G$ point transformations taking solutions into solutions is known. Its corresponding Lie symmetry algebra $\mathcal{L}$ has dimension $r$ and is realized by basis of vector fields of the form

$$
\hat{X}_{a}=\sum_{j=1}^{q}\left(\phi_{a}^{j}(\mathrm{x}, \mathrm{u})-\sum_{\mu=1}^{p} \xi_{a}^{\mu}(\mathrm{x}, \mathrm{u}) \frac{\partial u_{j}}{\partial x_{\mu}}\right) \partial_{u_{j}}, \quad a=1, \ldots, r .
$$

Each vector field $\hat{X}_{a}$ has the property that its $n$th prolongation annihilates the system (1.1) on its solution set:

$$
\left.\operatorname{pr}^{(k)}(\hat{X}) \Delta^{l}\right|_{\Delta^{n}=0}=0, \quad l, n=1, \ldots, m .
$$


The functions $\xi_{a}^{\mu}$ and $\phi_{a}^{j}$ are thus explicitly known. There exists standard algorithms for determining the symmetry algebra $\mathcal{L}$ and classifying subalgebras $\mathcal{L}_{p}$ of $\mathcal{L}$ : see refs. [5]-[7]. Let us consider a subgroup $G_{o} \subset G$ and its subalgebra $\mathcal{L}_{o} \subset \mathcal{L}$. A solution u $=f(\mathrm{x})$ of the system (1.1) is $G_{o^{-}}$-invariant if its graph $\Gamma_{f}=\{\mathrm{x}, f(\mathrm{x})\}$ is a $G_{o^{-}}$-invariant set:

$$
g \cdot \Gamma_{f}=\Gamma_{f}, \quad g \in G_{o}
$$

The matrix of characteristics of vector fields $\hat{X}_{a}$ which span the consider subalgebra $\mathcal{L}_{o}$ $\left(\operatorname{dim}\left[\mathcal{L}_{o}\right]=r_{o}\right)$ is defined by:

$$
Q_{a}^{j}\left(\mathrm{x}, \mathrm{u}^{(1)}\right)=\left\{\hat{X}_{a} u_{j}\right\}, \quad a=1, \ldots, r_{o}, \quad j=1, \ldots, q .
$$

By solving simultaneously system (1.1) and the $q \times r_{o}$ characteristic equations $Q_{a}^{j}\left(\mathrm{x}, \mathrm{u}^{(1)}\right)=$ 0 ; i.e. $\operatorname{rank}[\mathrm{Q}]=0$, associated with the basis elements of the Lie algebra $\mathcal{L}_{o}$, we obtain $G_{o}$-invariant solutions of the original system (1.1). The procedures to compute such type of solutions, known as the "classical" symmetry reduction method (SRM), is explained in greater details in refs. [1]-[5], which also provide numerous examples of such calculations.

Now, let us introduce the ideal magnetohydrodynamic (MHD) equations which describe nonrelativistic isentropic flows of an ideal (dissipative effects are negligible) and conductive (an infinite electrical conductivity is supposed) fluid, they have the following form [8]:

$$
\begin{aligned}
\frac{\mathrm{d} \rho}{\mathrm{d} t}+(\nabla \cdot \mathbf{v}) \rho & =0, \\
\frac{\mathrm{d} \mathbf{v}}{\mathrm{d} t}+\rho^{-1} \nabla p+\rho^{-1} \mathbf{B} \times(\nabla \times \mathbf{B}) & =\mathbf{0}, \\
\frac{\mathrm{d} p}{\mathrm{~d} t}+\gamma(\nabla \cdot \mathbf{v}) p & =0, \\
\frac{\partial \mathbf{B}}{\partial t}-\nabla \times(\mathbf{v} \times \mathbf{B}) & =\mathbf{0}, \\
\nabla \cdot \mathbf{B} & =0,
\end{aligned}
$$

where $\mathrm{d} / \mathrm{d} t=\partial / \partial t+(\mathbf{v} \cdot \nabla)$ is the convective derivative. Here $\rho$ is the mass density, $p$ is the pressure, $\mathbf{v}=\left(\mathrm{v}_{1}, \mathrm{v}_{2}, \mathrm{v}_{3}\right)$ and $\mathbf{B}=\left(\mathrm{B}_{1}, \mathrm{~B}_{2}, \mathrm{~B}_{3}\right)$ are the flow velocity and the magnetic field, respectively, and $\gamma$ is the adiabatic exponent. The MHD system (1.6) represents a quasilinear hyperbolic system, written in the Cauchy-Kowalewski form, of nine PDEs involving eight dependent variables $\mathrm{u}=(\rho, p, \mathbf{v}, \mathbf{B}) \in \mathbb{R}^{8}$ which depend on four independent variables denoted by $\mathrm{x}=(x, y, z, t) \in \mathbb{R}^{4}$.

A detailed group analysis of the MHD system (1.6) has been investigated by Fuchs and Richter [9], and independently by [10]. The largest Lie symmetry algebra of eqs.(1.6) is spanned by 13 infinitesimal generators (according to summation convention):

$$
\begin{aligned}
P_{\mu} & =\partial_{x_{\mu}}, \quad L_{k}=\epsilon_{k i j}\left(x_{i} \partial_{x_{j}}+\mathrm{v}_{i} \partial_{\mathrm{v}_{j}}+\mathrm{B}_{i} \partial_{\mathrm{B}_{j}}\right), \quad K_{i}=t \partial_{x_{i}}+\partial_{\mathrm{v}_{i}}, \\
F & =t \partial_{t}+x_{i} \partial_{x_{i}}, \quad G=-t \partial_{t}-2 \rho \partial_{\rho}+\mathrm{v}_{i} \partial_{\mathrm{v}_{i}}, \quad H=2 \rho \partial_{\rho}+2 p \partial_{p}+\mathrm{B}_{i} \partial_{\mathrm{B}_{i}},
\end{aligned}
$$

where $\epsilon_{k i j}$ is the Levi-Civita symbol, $i, j, k=1,2,3 ; \mu=0,1,2,3$. Thus, the MHD system is invariant under time $\left(P_{o}\right)$ and spatial $\left(P_{i}\right)$ translations, rotations $\left(L_{k}\right)$, Galilei transformations $\left(K_{i}\right)$, and dilations $(F, G$, and $H)$. In contrast to the results obtained for the $(1+1)$ and $(2+1)$ dimensional versions of the MHD model [9], the dimension of 
the Lie algebra for eqs. (1.6) in the full $(3+1)$ dimensions is independent of the value of the adiabatic exponent $\gamma$ that we treat here as a fixed parameter: $\gamma \geq 1$. A complete and exhaustive classification by conjugacy classes of $r$-dimensional subalgebras $(1 \leq r \leq 4)$ of this symmetry algebra has been established by [10]. Some examples of $G$-invariants solutions of eqs. (1.6) obtained from the SRM can be found in refs. [11] and [12].

There exists several alternative reduction procedures which go beyond Lie's classical methods and thus producing further solutions. They all have in common that they add some system of equations to the original system (1.1); the differential constraints, and that the extended system is solve simultaneously. These additional constraints replace the characteristic system $\operatorname{rank}[Q]=0$ of the classical SRM. Notice that a broad review of recent developments on this subject can be found in ref. [13].

The aim of this paper was to obtain some exact analytic solutions of eqs. (1.6) from the weak transversality method. In the next section, we will see that this alternative approach was particularly efficient to compute some MHD spherically invariant solutions.

\section{Spherically invariant solutions}

In this section, we apply the weak transversality method (WTM) based on the concept of "Group invariant solutions without transversality" [14] to determine some spherical invariant solutions of the MHD system (1.6). We begin by giving some basic theoretical notions of the WTM applied to a system of PDEs (1.1). Next, we present three examples illustrating the calculations involving the WTM. To motivate our calculations better, we present a detail description of the procedure for constructing such solutions.

Let us introduce the matrices $\Omega_{1}$ and $\Omega_{2}$ of the coefficients of vector fields (1.2):

$$
\Omega_{1}=\left\{\xi_{a}^{\mu}(\mathrm{x}, \mathrm{u})\right\}, \quad \Omega_{2}=\left\{\xi_{a}^{\mu}(\mathrm{x}, \mathrm{u}), \phi_{a}^{j}(\mathrm{x}, \mathrm{u})\right\}, \quad \Omega_{1} \in \mathbb{R}^{r \times p}, \quad \Omega_{2} \in \mathbb{R}^{r \times(p+q)} .
$$

Next, we take a specific subalgebra $\mathcal{L}_{o} \subset \mathcal{L}$ from which we use to calculate some group invariant solutions of (1.1). If the corresponding subgroup $G_{o}$ acts regularly and transversally on the manifold $\mathcal{M}=E \times U$ then

$$
\operatorname{rank}\left[\Omega_{1}\right]=\operatorname{rank}\left[\Omega_{2}\right] .
$$

This rank condition (2.2) is called strong transversality condition, and is equal to the generic orbit of $G_{o}$ on $\mathcal{M}$. This means that for each point $(\mathrm{x}, \mathrm{u}) \in \mathcal{M}$ where relation (2.2) holds, the classical SRM can be applied: i.e. the rank of the Jacobian matrix is maximal (see Chap. 3.5 of ref. [1]). If condition (2.2) is not satisfied, Anderson et al. [14] developed a method from which it is possible to calculate $G_{o}$-invariant solutions under certain conditions on $G_{O} \subset G$. Moreover, their novel approach can be simplified by introducing the concept of weak transversality: condition (2.2) is said to be satisfied in the weak sense if it holds only on a certain domain $\mathcal{M}_{o} \subset \mathcal{M}$ rather than on the entire space $\mathcal{M}$. It was shown in ref. [13] that subalgebras $\mathcal{L}_{o} \subset \mathcal{L}$ with weak transversality can still be used to construct invariant solutions using a specific algorithm which constitutes the WTM. In order to do it, we determine the class $S$ of functions u $=f(\mathrm{x})$ satisfying relation (2.2), next we require that the condition $\operatorname{rank}[Q]=0$ is satisfied on the set $S$, finally by substituting these obtained expressions into eqs. (1.1) and solving the corresponding reduced system, then we get some $G_{O^{-}}$invariant solutions of the original system (1.1). 
Example 1. We begin with the subalgebra $\mathcal{L}_{1}=\left\{L_{1}, L_{2}, L_{3}\right\}$.

The corresponding invariants of the subalgebra $\mathcal{L}_{1}$ are

$$
\begin{aligned}
& t, r=\sqrt{x^{2}+y^{2}+z^{2}}, \quad \Phi_{1}=\sqrt{\mathrm{v}_{1}^{2}+\mathrm{v}_{2}^{2}+\mathrm{v}_{3}^{2}}, \quad \Phi_{2}=x \mathrm{v}_{1}+y \mathrm{v}_{2}+z \mathrm{v}_{3}, \\
& \Phi_{3}=\sqrt{\mathrm{B}_{1}^{2}+\mathrm{B}_{2}^{2}+\mathrm{B}_{3}^{2}}, \quad \Phi_{4}=x \mathrm{~B}_{1}+y \mathrm{~B}_{2}+z \mathrm{~B}_{3}, \quad \Phi_{5}=\rho, \quad \Phi_{6}=p .
\end{aligned}
$$

Note that the condition on the Jacobian matrix

$$
\operatorname{rank}\left(\frac{\partial \Phi_{i}}{\partial \mathrm{u}_{j}}\right)=7, \quad i=1, \ldots, 6, \quad j=1, \ldots, 8,
$$

implies that the dimension of the graph of the solution $\Gamma_{f}$ is not preserved by the action of the symmetry group $G_{o}$. The graph of the solution sweeps out an orbit of dimension $\operatorname{dim}\left(G_{o} \Gamma_{f}\right)=p+\delta=5$. This is also due to the fact that the defect structure $\delta$ of the solution with respect the group $G_{1}$ and $\operatorname{rank}[Q]$ are both equal to one.

Therefore, we cannot obtain $G_{o}$-invariant solutions from this set of invariants by the classical symmetry reduction method, but only PIS relative to subalgebra $\mathcal{L}_{1}$. The equations determining the orbit of the graph of the solution are given by

$$
\begin{aligned}
\rho & =R(r, t), \quad p=A(r, t), \quad \mathrm{v}_{2}=\frac{\left(V-x \mathrm{v}_{1}\right)-z \mathrm{v}_{3}}{y}, \\
\mathrm{v}_{3} & =\frac{z\left(V-x \mathrm{v}_{1}\right)+\varepsilon y \sqrt{\left[U^{2}-\mathrm{v}_{1}^{2}\right]\left(y^{2}+z^{2}\right)-\left(x \mathrm{v}_{1}-V\right)^{2}}}{y^{2}+z^{2}}, \quad \mathrm{~B}_{2}=\frac{\left(X-x \mathrm{~B}_{1}\right)-z \mathrm{~B}_{3}}{y^{2}+z^{2}}, \\
\mathrm{~B}_{3} & =\frac{z\left(Y-x \mathrm{~B}_{1}\right)+\varepsilon y \sqrt{\left[X^{2}-\mathrm{B}_{1}^{2}\right]\left(y^{2}+z^{2}\right)-\left(x \mathrm{~B}_{1}-Y\right)^{2}}}{y^{2}+z^{2}}, \quad \varepsilon= \pm 1, \quad
\end{aligned}
$$

where $\Phi^{1}=U(r, t), \Phi^{2}=V(r, t), \Phi^{3}=X(r, t), \Phi^{4}=Y(r, t), \Phi^{5}=R(r, t)$ and $\Phi^{6}=$ $A(r, t)$ are arbitrary functions of their arguments. Note that no constraint have been imposed on functions $\mathrm{v}_{1}$ and $\mathrm{B}_{1}$; so they are arbitrary functions of $(x, y, z, t)$.

Substituting expressions (2.5) into eqs. (1.6) gives a reduced system which leads us to many calculations involving nonlinear equations. To compute the solutions of this system, we have to satisfy the compatibility conditions provided by the algorithm presented in ref. [5]. Thus the solution will be a nonreducible PIS because $\mathcal{L}_{1}$ does not contain any two dimensional subalgebra. We will not carry on these calculations here, but we will show that we can obtain solutions in a more effective way with the WTM.

The matrices $\Omega_{1}=\left\{\xi_{a}^{\mu}(\mathrm{x}, \mathrm{u})\right\}$ and $\Omega_{2}=\left\{\xi_{a}^{\mu}(\mathrm{x}, \mathrm{u}), \phi_{a}^{j}(\mathrm{x}, \mathrm{u})\right\}$ are represented by

$$
\Omega_{1}=\left(\begin{array}{ccc}
0 & z & -y \\
-z & 0 & x \\
y & -x & 0
\end{array}\right), \quad \Omega_{2}=\left(\begin{array}{ccccccccc}
0 & z & -y & 0 & \mathrm{v}_{3} & -\mathrm{v}_{2} & 0 & \mathrm{~B}_{3} & -\mathrm{B}_{2} \\
-z & 0 & x & -\mathrm{v}_{3} & 0 & \mathrm{v}_{1} & -\mathrm{B}_{3} & 0 & \mathrm{~B}_{1} \\
y & -x & 0 & \mathrm{v}_{2} & -\mathrm{v}_{1} & 0 & \mathrm{~B}_{2} & -\mathrm{B}_{1} & 0
\end{array}\right) .
$$

We observe that for the subalgebra $\mathcal{L}_{1}$ the transversality condition $(2.2)$ is not satisfied since the matrix $\Omega_{1}$ has rank 2 , while the matrix $\Omega_{2}$ has rank 3 . In this situation, the transversality condition is violated in the strong sense, and in principle the classical SRM cannot be applied here because the Jacobian matrix will not have maximal rank. However, we can force the rank of the matrix $\Omega_{2}$ to be equal to 2 , which is equivalent to a system of algebraic equations for components of $\mathbf{v}$ and $\mathbf{B}$, this is obtained by requiring that the 
determinants of all $3 \times 3$ matrices constructed using the rows and columns of $\Omega_{2}$ to equal zero. Solving this algebraic system, we obtain the class $S$ of functions $\mathbf{v}=\left(\mathrm{v}_{1}, \mathrm{v}_{2}, \mathrm{v}_{3}\right)$ and $\mathbf{B}=\left(\mathrm{B}_{1}, \mathrm{~B}_{2}, \mathrm{~B}_{3}\right)$ governed by the conditions:

$$
\rho=\rho(x, y, z, t), \quad p=p(x, y, z, t), \quad \mathbf{v}=f(x, y, z, t) \mathbf{r}, \quad \mathbf{B}=g(x, y, z, t) \mathbf{r},
$$

where $\mathbf{r}=(x, y, z)$ is the position vector, on these conditions there exists a certain domain $\mathcal{D}$ where the rank condition is fulfilled (transversality is weakly restored), i.e.,

$$
\left.\operatorname{rank}\left[\Omega_{1}\right]\right|_{\mathcal{D}}=\left.\operatorname{rank}\left[\Omega_{2}\right]\right|_{\mathcal{D}}
$$

As second step, we solve the characteristic system $\operatorname{rank}[Q]=0$ for the class $S$ of relations (2.6) which imposes the form of the functions $\rho, p, f$ and $g$ :

$$
\rho=R(r, t), \quad p=A(r, t), \quad f=f(r, t), \quad g=g(r, t),
$$

where $r=\sqrt{x^{2}+y^{2}+z^{2}}$. So the subgroup $G_{1}$ acts regularly and transversally. Consequently, invariant solutions can be constructed [13]. It is noteworthy to remark that

$$
L_{i}(\rho-R(r, t))=0, \quad L_{i}(p-A(r, t))=0, \quad L_{i}(\mathbf{v}-f(r, t) \mathbf{r})=0, \quad L_{i}(\mathbf{B}-g(r, t) \mathbf{r})=0,
$$

with $i=1,2,3$. Thus, functions (2.8) are invariant with respect to algebra $\mathcal{L}_{1}$ and satisfies weak (not strong) transversality. Relations (2.8) represent the most general form for the functions $\rho, p, \mathbf{v}$ and $\mathbf{B}$ to be rotationally invariant. Substituting these expressions for $\rho$, $p, \mathbf{v}$ and $\mathbf{B}$ into eqs. (1.6) then we get the corresponding reduced system:

$$
\begin{aligned}
\frac{\partial R}{\partial t}+r f \frac{\partial R}{\partial r}+\left(3 f+r \frac{\partial f}{\partial r}\right) R & =0, \\
\frac{\partial f}{\partial t}+\left(f+r \frac{\partial f}{\partial r}\right) f+\frac{1}{r R} \frac{\partial A}{\partial r} & =0, \\
\frac{\partial A}{\partial t}+r f \frac{\partial A}{\partial r}+\gamma\left(3 f+r \frac{\partial f}{\partial r}\right) A & =0, \\
\frac{\partial g}{\partial t}+r f \frac{\partial g}{\partial r}+3 f g & =0, \\
3 g+r \frac{\partial g}{\partial r} & =0 .
\end{aligned}
$$

From equations (2.12) and (2.13) we immediately find that

$$
\mathbf{B}=\frac{\mathcal{H}_{o}}{r^{3}} \mathbf{r}, \quad \mathcal{H}_{o} \in \mathbb{R} .
$$

We observe that the magnetic field $\mathbf{B}$ is static and potential; $\nabla \times \mathbf{B}=\mathbf{0}$. Thus, according to the Ampère's law [8] the current density is zero. So, the searched solutions describe a nonstationary and irrotational $\mathbf{B}$-aligned flow of a force-free fluid with spherical symmetry. By virtue of Kelvin's theorem [15], the circulation

$$
\Gamma_{\mathrm{c}}=\oint_{C} \mathbf{v} \cdot \mathrm{d} \boldsymbol{l}
$$


around a fluid element is always zero. The MHD system (1.6) is thus reduced to eqs. (2.9) and (2.11) that we solve by using the method of separation of variables. Next, we suppose that the flow is either incompressible or compressible.

Incompressible flow. Under the condition $\nabla \cdot \mathbf{v}=0$, we obtain two cases.

Case 1). The density $\rho=R_{o}$ is an arbitrary constant $\left(R_{o} \in \mathbb{R}^{+}\right)$:

$$
p=R_{o} \frac{\dot{a}}{r}-\frac{R_{o}}{2} \frac{a^{2}}{r^{4}}+b(t), \quad \mathbf{v}=\frac{a}{r^{3}} \mathbf{r}, \quad \mathbf{B}=\frac{\mathcal{H}_{o}}{r^{3}} \mathbf{r},
$$

where $\mathcal{H}_{o} \in \mathbb{R} ; a$ and $b$ are arbitrary functions of time (dots mean time derivatives).

Case 2). The density has the form $\rho=\rho(r, t)$ but with condition that $\mathrm{d} \rho / \mathrm{d} t=0$ :

$$
\begin{aligned}
\rho= & R_{o} \beta^{3 \lambda_{o}} \exp \left[-\lambda_{o} r^{3}\right], \quad \mathbf{v}=\frac{\dot{\beta}}{\beta} \mathbf{r}, \quad \mathbf{B}=\frac{\mathcal{H}_{o}}{r^{3}} \mathbf{r}, \\
p= & \alpha(t)+R_{o} \beta^{3 \lambda_{o}}\left[\left(\frac{3 \lambda_{o}}{2}+1-\frac{1}{2 r^{3}}\right)\left(\frac{\dot{\beta}}{\beta}\right)^{2}+\frac{\ddot{\beta}}{\beta}\right] \exp \left[-\lambda_{o} r^{3}\right] \\
& -\lambda_{o}{ }^{1 / 3} R_{o} \beta^{3 \lambda_{o}}\left[\left(\frac{3 \lambda_{o}}{2}-1\right)\left(\frac{\dot{\beta}}{\beta}\right)^{2}+\frac{\ddot{\beta}}{\beta}\right] \Gamma\left(\frac{2}{3}, \lambda_{o} r^{3}\right),
\end{aligned}
$$

where $R_{o}>0, \lambda_{o}$ and $\mathcal{H}_{o} \in \mathbb{R} ; \alpha$ and $\beta$ are arbitrary functions of time, and $\Gamma$ denotes the incomplete gamma function.

Compressible flow. From eqs. (2.9) and (2.11), we have that $p=A_{o} \rho^{\gamma}\left(A_{o} \in \mathbb{R}^{+}\right)$. We solved the system composed of eqs. (2.9) and (2.10) by considering the following cases.

Case 1). We impose that $f=f(t)$, and then obtain this MHD spherical solution:

$$
\begin{aligned}
& \rho=\left\{\begin{array}{l}
R_{o} \exp \left[-3 \int^{t} f\left(t^{\prime}\right) d t^{\prime}-\left(\dot{f}+f^{2}\right) \frac{r^{2}}{2}\right] \quad \text { for } \quad \gamma=1, \\
\left(\frac{\gamma-1}{\gamma A_{0}}\right)^{1 /(\gamma-1)}\left[C_{1} \exp \left[3(\gamma-1) \int^{t} f\left(t^{\prime}\right) d t^{\prime}\right]-\left(\dot{f}+f^{2}\right) \frac{r^{2}}{2}\right]^{1 /(\gamma-1)} \text { for } \gamma>1,
\end{array}\right. \\
& p=A_{o} \rho^{\gamma}, \quad \mathbf{v}=f(t) \mathbf{r}, \quad \mathbf{B}=\frac{\mathcal{H}_{o}}{r^{3}} \mathbf{r},
\end{aligned}
$$

where $R_{o}, A_{o}, C_{1}$ and $\mathcal{H}_{o}$ are arbitrary constants $\left(R_{o}, A_{o} \in \mathbb{R}^{+}\right)$. The function $f$ satisfies:

$$
\ddot{f}+(3 \gamma+1) f \dot{f}+(3 \gamma-1) f^{3}=0,
$$

which can be solved as follows. First, we introduce the change of variable

$$
\zeta(f)=\dot{f},
$$

so that the ordinary differential equations (ODE) (2.19) can be rewritten as

$$
\zeta \frac{\mathrm{d} \zeta}{\mathrm{d} f}+(3 \gamma+1) f \zeta+(3 \gamma-1) f^{3}=0 .
$$

Using the second change of variable

$$
\zeta(f)=f^{2} \chi(\tau), \quad \text { where } \quad \tau=\ln f,
$$


which transforms equation (2.21) into

$$
\chi \frac{\mathrm{d} \chi}{\mathrm{d} \tau}+2 \chi^{2}+(3 \gamma+1) \chi+(3 \gamma-1)=0
$$

that we solve to finally get

$$
\tau=-\int \frac{\chi d \chi}{2 \chi^{2}+(3 \gamma+1) \chi+(3 \gamma-1)}+\tau_{o}, \quad \tau_{o} \in \mathbb{R} .
$$

It is noteworthy to mention that ODE (2.19) admits this particular solution

$$
f(t)=\frac{1}{\left(\lambda_{o} t+t_{o}\right)}, \quad \lambda_{o}=1,3 \gamma / 2, \quad t_{o} \in \mathbb{R},
$$

from which we get the following MHD spherical solution:

$$
\begin{aligned}
& \rho=\left\{\begin{array}{l}
\frac{R_{o}}{\left(\lambda_{o} t+t_{o}\right)^{3}} \exp \left[\frac{\left(\lambda_{o}-1\right)}{\left(\lambda_{o} t+t_{o}\right)^{2}} \frac{r^{2}}{2}\right] \quad \text { for } \quad \gamma=1, \\
\left(\frac{\gamma-1}{\gamma A_{0}}\right)^{1 /(\gamma-1)}\left(C_{1}\left(\lambda_{o} t+t_{o}\right)^{3(1-\gamma) / \lambda_{o}}+\frac{\left(\lambda_{o}-1\right)}{\left(\lambda_{o} t+t_{o}\right)} \frac{r^{2}}{2}\right)^{1 /(\gamma-1)} \text { for } \gamma>1
\end{array}\right. \\
& p=A_{o} \rho^{\gamma}, \quad \mathbf{r}=\frac{\mathbf{r}}{\left(\lambda_{o} t+t_{o}\right)}, \quad \mathbf{B}=\frac{\mathcal{H}_{o}}{r^{3}} \mathbf{r},
\end{aligned}
$$

where $R_{o}>0, A_{o}>0, t_{o}, C_{1}, \mathcal{H}_{o}$ are arbitrary constants. Note that for solution (2.26), the radial profiles of density and pressures are similar to the ones obtained in [16] for a purely rotating flow of a force-free compressible fluid with finite pressure. However, in our present case the flow is nonstationary and irrotational.

Case 2). Now, we consider the case $f=f(r, t)$ that gives us two types of solutions which depend on the values of the adiabatic exponent $\gamma$.

1). For the isothermal case $\gamma=1$ :

$$
\rho=R_{o} \exp \left[-\lambda_{o} t-\frac{\eta^{2}}{2 A_{o}}\right], \quad p=A_{o} \rho, \quad \mathrm{v}=\frac{\eta(r)}{r} \mathbf{r}, \quad \mathbf{B}=\frac{\mathcal{H}_{o}}{r^{3}} \mathbf{r},
$$

where $R_{o}, A_{o}$, and $\lambda_{o} \in \mathbb{R}^{+} /\{0\}, \mathcal{H}_{o} \in \mathbb{R}$. The function $\eta$ solves this first order ODE

$$
\left(1-\frac{\eta^{2}}{2 A_{o}}\right) \eta^{\prime}+\frac{2}{r} \eta-\lambda_{o}=0
$$

where the prime' denotes differentiation with respect to $r$.

Equation (2.28) does not have the Painlevé property for any values of $A_{o}$ and $\lambda_{o}$. If such an ODE has the Painlevé property means that its general solution has no movable singularities other than poles (i.e. essential singularities or branch points), then it can be transformed into one of transcendent forms [17] and integrated in terms of some known functions. The test verifying whether a given ODE satisfies certain necessary conditions for having the Painlevé property, is algorithmic [18] and can be performed using a specifically written MATHEMATICA program [19]. Thus, the ODE (2.28) is difficult to integrate, and we have not able to find an explicit solution of it. We must look for a solution by numerical methods, and this task would take us beyond the scope of this paper. 
2). $\quad \gamma>1$. The reductions lead us to the following MHD spherical solution:

$$
\rho=\frac{u^{\prime}(r)}{r^{2} u(r)}\left(\lambda_{o} t+t_{o}\right)^{-\kappa}, \quad p=A_{o} \rho^{\gamma}, \quad \mathbf{v}=\frac{u(r)}{r u^{\prime}(r)} \frac{\mathbf{r}}{\left(\lambda_{o} t+t_{o}\right)}, \quad \mathbf{B}=\frac{\mathcal{H}_{o}}{r^{3}} \mathbf{r},
$$

where $A_{o}, \lambda_{o}, t_{o}$ and $\mathcal{H}_{o}$ are arbitrary constants, and $\kappa=2 /(1-\gamma)$. The unknown function $u(r)$ is determined by solving the second order ODE:

$$
\begin{aligned}
{\left[\gamma A_{o} r^{-4 / \kappa}\left(u^{\prime}\right)^{2(\kappa+1) / \kappa}-u^{2}\right] } & u^{\prime \prime}+\gamma A_{o}\left(\kappa \lambda_{o}-1\right) r^{-4 / \kappa}\left(u^{\prime}\right)^{2(2 \kappa+1) / \kappa} u^{\left[2\left(\lambda_{o}-1\right) \kappa-2\right] / \kappa} \\
& -2 r^{(\kappa+4) / \kappa}\left(u^{\prime}\right)^{(3 \kappa+2) / \kappa} u^{2\left(\kappa \lambda_{o}-1\right) / \kappa}+\left(\lambda_{o}-1\right)\left(u^{\prime}\right)^{2} u=0,
\end{aligned}
$$

with the constraint that $u^{\prime}(r) /\left(r^{2} u(r)\right)>0$ in order to get $\rho>0$. Equation (2.30) does not have the Painlevé property. In fact, a necessary condition for this ODE to have the Painlevé property is that it is expressible in the form

$$
f^{\prime \prime}=L(r, f)\left(f^{\prime}\right)^{2}+M(r, f)\left(f^{\prime}\right)+N(r, f),
$$

where $L, M$ and $N$ are rational in $f$, with coefficients analytical in $r$ (see page 321 of [17]). But for all values of $\kappa$ and $\lambda_{o}$, the ODE (2.30) cannot be written in that form.

Next, we present two other examples of spherical invariant solutions of the MHD equations (1.6) obtained from four-dimensional subalgebras taken from [10]. Such solutions are determined in the same manner as explained in example 1.

Example 2. Let us consider the subalgebra $\mathcal{L}_{2}=\left\{L_{1}, L_{2}, L_{3}, P_{o}\right\}$. The matrices $\Omega_{1}$ and $\Omega_{2}$ take the form

$$
\Omega_{1}=\left(\begin{array}{cccc}
0 & z & -y & 0 \\
-z & 0 & x & 0 \\
y & -x & 0 & 0 \\
0 & 0 & 0 & 1
\end{array}\right), \Omega_{2}=\left(\begin{array}{cccccccccc}
0 & z & -y & 0 & 0 & \mathrm{v}_{3} & -\mathrm{v}_{2} & 0 & \mathrm{~B}_{3} & -\mathrm{B}_{2} \\
-z & 0 & x & 0 & -\mathrm{v}_{3} & 0 & \mathrm{v}_{1} & -\mathrm{B}_{3} & 0 & \mathrm{~B}_{1} \\
y & -x & 0 & 0 & \mathrm{v}_{2} & -\mathrm{v}_{1} & 0 & \mathrm{~B}_{2} & -\mathrm{B}_{1} & 0 \\
0 & 0 & 0 & 1 & 0 & 0 & 0 & 0 & 0 & 0
\end{array}\right) .
$$

It is immediate to check that the local transversality is violated for this algebra in the strong sense since $\operatorname{rank}\left[\Omega_{1}\right]=3$ and $\operatorname{rank}\left[\Omega_{2}\right]=4$. By requiring that matrix $\Omega_{2}$ should have rank 3, we reobtain constraints (2.6). Next, we solve the characteristic system $\operatorname{rank}[Q]=0$, and then get the form of the searched solution:

$$
\rho=R(r), \quad p=A(r), \quad \mathbf{v}=f(r) \mathbf{r}, \quad \mathbf{B}=g(r) \mathbf{r},
$$

which corresponds to the stationary form of functions (2.8). Now, by substituting expressions (2.32) into eqs. (1.6), we find these relations:

$$
p=A_{o} \rho^{\gamma}, \quad \mathbf{B}=\frac{\mathcal{H}_{o}}{r^{3}} \mathbf{r},
$$

where $A_{o}>0, \mathcal{H}_{o} \in \mathbb{R}$, and get this reduced system of ODEs:

$$
\begin{aligned}
r f R^{\prime}+\left(3 f+r f^{\prime}\right) R & =0, \\
r f f^{\prime}+f^{2}+\frac{A_{o}}{r} R^{(\gamma-2)} R^{\prime} & =0 .
\end{aligned}
$$


From equation (2.34) we get the expression for the density

$$
R=\frac{R_{o}}{r^{3} f},
$$

where $R_{o} \in \mathbb{R}$. Substituting relation (2.36) into ODE (2.35) and then integrating it, we obtain an algebraic equation which determines the function $f$ :

$$
\begin{array}{r}
r^{2} f^{2}+2 A_{o} \ln \left[\frac{R_{o}}{r^{3} f}\right]=C_{o} \quad \text { for } \quad \gamma=1, \\
r^{2} f^{2}+\frac{2 \gamma A_{o}}{(\gamma-1)} R_{o}^{(\gamma-1)} r^{3(1-\gamma)} f^{(1-\gamma)}=C_{o} \quad \text { for } \quad \gamma>1 .
\end{array}
$$

This case represents a stationary rotationally invariant solution of the MHD system (1.6). Notice that relation (2.37) corresponds to the well known Bernoulli's equation [15]:

$$
\frac{1}{2}|\mathbf{v}|^{2}+\int \frac{\mathrm{d} p}{\rho}=\text { constant. }
$$

Here, the streamlines coincide with the lines of force. As a consequence

$$
(\mathbf{v} \cdot \nabla)\left(\frac{1}{2}|\mathbf{v}|^{2}+\int \frac{\mathrm{d} p}{\rho}\right)=0,
$$

which means that Bernoulli's equation is still valid along the streamlines. The relation $\nabla \rho \times \mathbf{B}=\mathbf{0}$ indicates that the mass density gradient is along the flow. We retrieve here magnetized nozzle configuration [16] in the context of a spherical geometry.

Example 3. Finally, we conclude this investigation by considering the subalgebra $\mathcal{L}_{3}=\left\{L_{1}, L_{2}, L_{3}, F+G+\alpha H\right\}$, with $\alpha \in \mathbb{R}$. The matrices $\Omega_{1}$ and $\Omega_{2}$ are given by

$$
\begin{aligned}
\Omega_{1} & =\left(\begin{array}{ccc}
0 & z & -y \\
-z & 0 & x \\
y & -x & 0 \\
x & y & z
\end{array}\right), \\
\Omega_{2} & =\left(\begin{array}{cccccccccccc}
0 & z & -y & 0 & 0 & 0 & \mathrm{v}_{3} & -\mathrm{v}_{2} & 0 & \mathrm{~B}_{3} & -\mathrm{B}_{2} \\
-z & 0 & x & 0 & 0 & -\mathrm{v}_{3} & 0 & \mathrm{v}_{1} & -\mathrm{B}_{3} & 0 & \mathrm{~B}_{1} \\
y & -x & 0 & 0 & 0 & \mathrm{v}_{2} & -\mathrm{v}_{1} & 0 & \mathrm{~B}_{2} & -\mathrm{B}_{1} & 0 \\
x & y & z & 2 \alpha & 2(1+\alpha) & \mathrm{v}_{1} & \mathrm{v}_{2} & \mathrm{v}_{3} & \alpha \mathrm{B}_{1} & \alpha \mathrm{B}_{2} & \alpha \mathrm{B}_{3}
\end{array}\right) .
\end{aligned}
$$

The transversality condition (2.2) is again violated in the strong sense, because rank $\left[\Omega_{1}\right]=$ 3 and $\operatorname{rank}\left[\Omega_{2}\right]=4$. If we force the matrix $\Omega_{2}$ to be rank 3 , we still obtain the constraints (2.6). However, from the characteristic system $\operatorname{rank}[Q]=0$ we get here:

$$
\rho=R(t) r^{2 \alpha}, \quad p=A(t) r^{2(\alpha+1)}, \quad \mathbf{v}=f(t) \mathbf{r}, \quad \mathbf{B}=g(t) r^{(\alpha-1)} \mathbf{r},
$$

where $R, A, f$ and $g$ are arbitrary functions of time. Next, substituting relation (2.40) into eqs. (1.6), it follows that $\alpha=-2$ and then we obtain the following solution:

$$
\rho=\frac{R_{o}}{r^{4}} \xi(t), \quad p=\frac{A_{o}}{r^{2}} \xi^{(2-3 \gamma)}, \quad \mathbf{v}=\frac{\dot{\xi}}{\xi} \mathbf{r}, \quad \mathbf{B}=\frac{\mathcal{H}_{o}}{r^{3}} \mathbf{r},
$$


where $R_{o}$ and $A_{o} \in \mathbb{R}^{+} /\{0\}, \mathcal{H}_{o} \in \mathbb{R}$. The function $\xi$ solves this ODE:

$$
\ddot{\xi}-\frac{2 A_{o}}{R_{o}} \xi^{(2-3 \gamma)}=0 .
$$

Solution (2.41) represents another example of a nonstationary rotationally invariant solution: it describes a compressible and irrotational B-aligned flow of a force-free fluid. In general, the ODE (2.42) does not have Painlevé property. However, it does for special values of the parameter $\gamma$, namely $\gamma=-1 / 3$ and $\gamma=0$, but both cases are unphysical since $\gamma \geq 1$. So, the equation (2.42) must be solve numerically.

\section{Conclusion}

We summarize the main results achieved in this article. Using systematically the weak transversality method (WTM) based on a group theoretical framework, we found here some novel (at the best of our knowledge) spherical solutions of the MHD system (1.6) with specific physical properties. All solutions obtained here are rotationally invariant. As consequence, the flow is irrotational and collinear to a static and potential magnetic field $\mathbf{B}$, implying that the fluid is force-free. Notice that MHD spherical solutions are of physical interests in magnetic confinement theory [8], and force-free conditions are widely applicable in astrophysical environments because forces other than electromagnetic are comparatively much smaller [20]. For Example 1, we computed several types of spherical solutions which describe incompressible and compressible flows. Example 2 describes a stationary solution which corresponds to a magnetized nozzle configuration [16] in the context of a spherical geometry. Finally, for Example 3, we obtained another type of spherical solution which represents a nonstationary flow of a compressible fluid. So, to conclude, this work demonstrates the efficiency of the WTM for obtaining exact analytic solutions of systems of PDEs, especially the spherical ones of ideal MHD equations.

\section{References}

[1] Olver P J, Applications of Lie Groups to Differential Equations, Springer-Verlag, Berlin, 1993.

[2] Ovsiannikov L V, Group Analysis of Differential Equations, Academic Press, New York, 1982.

[3] Ibragimov N H, Elementary Lie Group Analysis and Ordinary Differential Equations, John Wiley \& Sons, Chichester, 1999.

[4] Hydon P E, Symmetry Methods for Differential Equations, Cambridge University Press, Cambridge, 2000.

[5] Clarkson P A, Winternitz P, Symmetry Reduction and Exact Solutions of Nonlinear Partial Differential Equations, Proceedings of The Painlevé Property One Century Later, Editor Conte R, Springer-Verlag, New York, 1999, Chapter 10, 597-668. 
[6] CRC Handbook on Lie Group Methods for Differential Equations, edited by N. H. Ibragimov, CRC Press, Boca Raton, Fl, 1994, Vol 1; ibid, CRC Press, Boca Raton, Fl, 1995, Vol 2; ibid, CRC Press, Boca Raton, Fl, 1996, Vol 3.

[7] Baumann G, Symmetry Analysis of Differential Equations with Mathematica, Springer, New York, 2000.

[8] Friedberg P, Ideal Magnetohydrodynamics, Plenum Press, New York, 1987.

[9] Fuchs J C, Richter W E, Similarity Solutions for the Two-dimensional Nonstationary Ideal MHD Equations, J. Math. Phys. A: Math. Gen. 20 (1987), 31353157 .

[10] Grundland A M, Lalague L, Lie Subgroups of Symmetry Groups of Fluid Dynamics and Magnetohydrodynamics Equations, Can. J. Phys. 73 (1995), 463-477.

[11] Fuchs J C, Symmetry Groups and Similarity Solutions of MHD Equations, J. Math. Phys. 32 (1991), 1703-1708.

[12] PICARD P Y, Some Exact solutions of the Ideal MHD Equations through Symmetry Reduction Method, J. Math. Anal. Appl. in press.

[13] Grundland A M, Tempesta P, Winternitz P, Weak Transversality and Partially Invariant Solutions, J. Math. Phys. 44 (2003), 2704-2722.

[14] Anderson I, Fels M, Torre C, Group Invariant Solutions without Transversality, Commun. Math. Phys. 212 (2000), 653-686.

[15] Batchelor G K, An Introduction to Fluid Dynamics, Cambridge University Press, Cambridge, 1967.

[16] Wang Z, Barnes C W, Exact Solutions to Magnetized Plasma Flow, Phys. Plasma 5, (2001) 957-963.

[17] Ince E L, Ordinary Differential Equations, Dover, New York, 1956.

[18] Ablowitz M J, Ramani A, Segur H, A Connection Between Nonlinear Evolution Equations and Ordinary Differential Equations of P-type, J. Math. Phys. 21 (1980), $715-721$.

[19] Baldwin D, Hereman W, Symbolic Software for the Painlevé Test of Nonlinear Ordinary and Partial Differential Equations, J. Nonlinear Math. Phys. 13 (2006), 90-110.

[20] Somov B V, Fundamentals of Cosmic Electrodynamics, Kluwer Academic Publishers, Dordrecht, 1994. 